\title{
Hydrodynamization in kinetic theory: Transient modes and the gradient expansion
}

\author{
Michal P. Heller, ${ }^{1,2,3,{ }^{*}}$ Aleksi Kurkela, ${ }^{4,5}{ }^{\dagger}$ Michał Spaliński, ${ }^{3,6, *}$ and Viktor Svensson ${ }^{3,1, \S}$ \\ ${ }^{1}$ Max Planck Institute for Gravitational Physics, Potsdam-Golm D-14476, Germany \\ ${ }^{2}$ Perimeter Institute for Theoretical Physics, Waterloo, Ontario N2L 2Y5, Canada \\ ${ }^{3}$ National Centre for Nuclear Research, 00-681 Warsaw, Poland \\ ${ }^{4}$ Theoretical Physics Department, CERN, CH-1211 Geneva, Switzerland \\ ${ }^{5}$ Faculty of Science and Technology, University of Stavanger, 4036 Stavanger, Norway \\ ${ }^{6}$ Physics Department, University of Biatystok, 15-245 Biatystok, Poland
}

(Received 17 November 2016; revised manuscript received 16 March 2018; published 31 May 2018)

\begin{abstract}
We explore the transition to hydrodynamics in a weakly coupled model of quark-gluon plasma given by kinetic theory in the relaxation-time approximation with conformal symmetry. We demonstrate that the gradient expansion in this model has a vanishing radius of convergence due to the presence of a transient (nonhydrodynamic) mode, in a way similar to results obtained earlier in strongly coupled gauge theories. This suggests that the mechanism by which hydrodynamic behavior emerges is the same, which we further corroborate by a novel comparison between solutions of different weakly and strongly coupled models. However, in contrast with other known cases, we find that not all the singularities of the analytic continuation of the Borel transform of the gradient expansion correspond to transient excitations of the microscopic system; some of them reflect analytic properties of the kinetic equation when the proper time is continued to complex values.
\end{abstract}

DOI: 10.1103/PhysRevD.97.091503

\section{INTRODUCTION AND SUMMARY}

Heavy-ion collisions at the Relativistic Heavy Ion Collider and LHC provide an outstanding opportunity to test our understanding of QCD. Perhaps unsurprisingly, a fully $a b$ initio theoretical description has turned out to be very challenging. This has led to the exploration of models of increasing complexity and often nonoverlapping domains of validity-see, e.g., Refs. [1,2] for a review of such models in the context of the hydrodynamic description in ultrarelativistic heavy-ion collisions.

In this paper, we focus on the poorly understood transient far-from-equilibrium regime, which precedes viscous hydrodynamic evolution of quark-gluon plasma (QGP). There are two approaches to the study of the transition to hydrodynamics (hydrodynamization) in non-Abelian gauge theories like QCD: a weakly coupled description based on effective kinetic theory (EKT) [3] (see also Refs. [4-9]) and a strongly coupled plasma paradigm based on

\footnotetext{
michal.p.heller@aei.mpg.de

†aleksi.kurkela@cern.ch

michal.spalinski@ncbj.gov.pl

viktor.svensson@aei.mpg.de
}

Published by the American Physical Society under the terms of the Creative Commons Attribution 4.0 International license. Further distribution of this work must maintain attribution to the author(s) and the published article's title, journal citation, and DOI. holography [10] (see Refs. [11-14] for sample results). They involve very different physical pictures and mathematical frameworks; the first relies on the Boltzmann equation, while the second makes use of higher-dimensional Einstein equations. Since under experimental conditions the QCD coupling is neither parametrically small nor large, it is crucial to understand which implications of these approaches can be viewed as universal.

Our aim is to shed light on equilibration in weakly coupled systems by examining large-order behavior of the hydrodynamic gradient expansion [15] in the framework of the kinetic theory model given by the Boltzmann equation in the relaxation-time approximation (RTA) [16]. We also assume conformal symmetry, as its breaking at strong coupling does not significantly alter equilibration processes [17,18], whereas at weak coupling at vanishing quark masses, it is a next-to-leading-order effect (see, e.g., Ref. [5]). The key result of this paper is demonstrating the vanishing radius of convergence of the gradient expansion in this kinetic theory model (see also Ref. [19] which studied in this context the RTA kinetic theory with constant relaxation time) and understanding some puzzling features revealed by these studies. The reason for the divergence turns out to be the same as in the case of holographic plasma: the presence of fast-decaying (nonhydrodynamic) modes [20-23] of which the relaxation controls the emergence of hydrodynamic behavior (and, in particular, its applicability to the physics of heavy-ion collisions [24-26]). This is connected with the existence of 
attractors that govern the evolution far from equilibrium [21,27-35]. In this context, see also Refs. [36,37] for studies of possible manifestations of analogous fast-decaying modes in trapped Fermi gases close to unitarity as well as Ref. [38], in which various features of transient modes are analyzed as a function of the microscopic interaction strength in a holographic toy model.

Quite remarkably, our analysis of the Borel transform of the gradient expansion in the RTA kinetic theory reveals not only the expected purely decaying mode [22] but also singularities that could naively be interpreted as transient contributions to the energy-momentum tensor exhibiting damped oscillatory behavior, similar to the findings of Ref. [39]. This would, however, be surprising, since the mechanism described there does not apply to the RTA theory [40]. In fact, we demonstrate below that these singularities are instead a manifestation of analytic properties of the evolution equations in complexified time. This feature is related to what has been observed in other contexts where large-order behavior of perturbative series expansions is used to draw conclusions about nonperturbative effects (see, e.g., Refs. [41-44]).

Our conclusions concerning hydrodynamization provide strong motivation for comparing numerical solutions of the RTA evolution equations with the EKT results at intermediate coupling reported in Refs. [8,9] and with the AdS/ CFT-based simulations of Ref. [45]. We uncover semiquantitative agreement, and as a byproduct of this analysis, we present a new and effective way of visualizing (see Fig. 2) the correlation between the hydrodynamization time and the value of the $\eta / s$ ratio noted in Ref. [46].

\section{KINETIC THEORY}

We address the issues discussed above in the context of Bjorken flow [47], which is conveniently formulated in (proper-time) rapidity coordinates $\tau-y$. They are related to Minkowski lab-frame coordinates $t-z$ by $t=\tau \cosh y$ and $z=\tau \sinh y$, where $z$ is the collision axis. Assuming translation symmetry in the transverse plane $\mathbf{x}_{T}$, the onshell distribution function $f$ depends only on the proper time $\tau$, the modulus of the transverse momentum $p \equiv\left|\mathbf{p}_{T}\right|$ and the boost-invariant variable $u=\tau^{2} p^{y}$.

In the RTA, the collision kernel appearing in the Boltzmann equation is linearized around the equilibrium distribution, which for simplicity we take to be Boltzmann,

$$
f_{0}(\tau, u, p)=\frac{1}{(2 \pi)^{3}} \exp \left[-\frac{\sqrt{u^{2}+p^{2} \tau^{2}}}{\tau T(\tau)}\right] .
$$

The RTA Boltzmann equation takes the form

$$
\frac{\partial f(\tau, u, p)}{\partial \tau}=\frac{1}{\tau_{\text {rel }}}\left\{f_{0}(\tau, u, p)-f(\tau, u, p)\right\} .
$$

To ensure conformal symmetry, we assume that the relaxation time is of the form

$$
\tau_{\text {rel }}=\frac{\gamma}{T(\tau)},
$$

where $\gamma$ is dimensionless and is the only parameter of this model. The dependence of temperature on the proper time is determined dynamically by imposing the Landau matching condition [48,49]

$$
\mathcal{E}(\tau)=\frac{3}{\pi^{2}} T^{4}(\tau)
$$

where

$$
\mathcal{E}(\tau)=2 \int d^{4} p \delta\left(p^{2}\right) \theta\left(p^{0}\right) \frac{u^{2}+p^{2} \tau^{2}}{\tau^{2}} f(\tau, u, p)
$$

is the energy density (per particle species).

\section{HYDRODYNAMIC GRADIENT EXPANSION}

In a conformal theory, the eigenvalues of the expectation value of the energy-momentum tensor in a boost-invariant state are functions of the proper time $\tau$ alone. They are given by the energy density $\mathcal{E}$ and the longitudinal and transverse pressures $\mathcal{P}_{L}$ and $\mathcal{P}_{T}$ :

$$
\mathcal{P}_{L}=-\mathcal{E}-\tau \dot{\mathcal{E}}, \quad \mathcal{P}_{T}=\mathcal{E}+\frac{1}{2} \tau \dot{\mathcal{E}} .
$$

Away from equilibrium, $\mathcal{P}_{L}$ and $\mathcal{P}_{T}$ differ from the equilibrium pressure at the same energy density $\mathcal{P} \equiv \mathcal{E} / 3$. It is convenient to study the approach to equilibrium by examining the behavior of the pressure anisotropy

$$
\mathcal{A} \equiv \frac{\mathcal{P}_{T}-\mathcal{P}_{L}}{\mathcal{P}}
$$

as a function of the dimensionless variable $w \equiv T \tau$. The gradient expansion of $\mathcal{A}$ takes the form

$$
\mathcal{A}(w)=\sum_{n=1}^{\infty} a_{n} w^{-n} .
$$

This follows directly from Eq. (6) and (7) if we use the fact that in conformal theories near equilibrium $\mathcal{E} \sim T^{4}$ and for boost-invariant flow $T \sim \tau^{-1 / 3}+O\left(\tau^{-1}\right)$ [47] (up to exponentially suppressed corrections).

To determine the coefficients $a_{n}$, we look for a solution of the Boltzmann equation (2) in the form

$f(\tau, u, p)=f_{0}(\tau, u, p)\left(1+\sum_{n=1}^{\infty} w^{-n} h_{n}\left(\frac{u}{w}, \frac{p}{T}\right)\right)$.

Inserting Eq. (9) into the Boltzmann equation (2), one can algebraically determine the functions $h_{n}$ in terms of the unknown coefficients $a_{n}$. A key step in doing this is to eliminate proper-time derivatives of temperature in favor of $\mathcal{A}$ and then using Eq. (8).

The Landau matching condition Eq. (4) implies that at each order $n>0$ 


$$
\int d^{4} p \delta\left(p^{2}\right) \theta\left(p^{0}\right) \frac{v^{2}}{\tau^{2}} f_{0}(\tau, u, p) h_{n}\left(\frac{u}{w}, \frac{p}{T}\right)=0 .
$$

This condition amounts to a linear, algebraic equation that determines the expansion coefficient $a_{n-1}$.

Proceeding this way, we have calculated the expansion coefficients analytically up to order 426 (we include the result of this calculation in the Supplemental Material [50]). As a cross-check, we have also calculated the first ten terms using two independent, albeit slower, methods from Refs. [48,49,51], noting perfect agreement.

The leading expansion coefficients read

$$
a_{1}=8 / 5 \gamma, \quad a_{2}=32 / 105 \gamma^{2}, \quad a_{3}=-416 / 525 \gamma^{3} .
$$

They can be used to match the transport coefficients of Baier-Romatschke-Son-Starinets-Stephanov hydrodynamics [20] to the RTA model. In particular, one finds

$$
\eta / s=\gamma / 5 \text {. }
$$

At large orders, the expansion coefficients in Eq. (8) exhibit factorial growth. This demonstrates the vanishing radius of convergence of the hydrodynamic series, which parallels similar findings obtained numerically in $\mathcal{N}=4$ super symmetric Yang-Mills theory using holography $[15,52]$ as well as in hydrodynamics $[21,53,54]$. This is to be expected on general grounds, since the RTA theory contains, apart from hydrodynamic excitations, also a shortlived mode [22] of which the physics is not captured by the truncated gradient expansion, as shown in Refs. [15,21].

\section{BOREL TRANSFORM AND SHORT-LIVED MODES}

In this section, we explore the Borel transform technique as a way to map out the excitations of expanding QGP.

The Borel transform removes the leading-order factorial growth of the coefficients,

$$
\mathcal{A}_{B}(\xi)=\sum_{n=1}^{\infty} \frac{a_{n}}{n !} \xi^{n}
$$

The inverse transform is given by the Borel summation formula

$$
\mathcal{A}_{\text {resummed }}(w)=\frac{1}{w} \int_{0}^{\infty} d \xi e^{-\xi / w} \mathcal{A}_{B}(\xi)
$$

and is not uniquely defined since the analytic continuation of $\mathcal{A}_{B}(\xi)$ necessarily contains singularities that are responsible for the vanishing radius of convergence of the original series. We analytically continue the series from Eq. (13) truncated at 426 terms by means of Padé approximants. Figure 1 shows the poles of the Padé approximant, which condense in a way known to signify a branch-point singularity [55].

In Ref. [21] (see also Refs. [15,52-54,56]), the ambiguity in the Borel summation associated with singularities

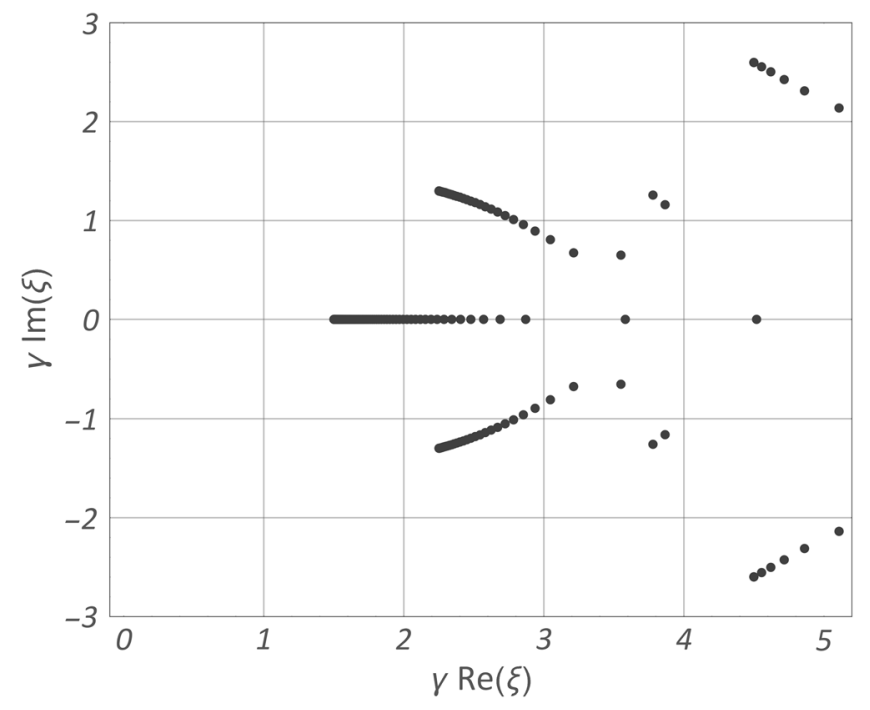

FIG. 1. Poles of the Padé approximant to the Borel transform of the gradient expansion. We have checked that the structure of singularities remains stable as the number of terms kept in the series is varied. We have also checked that the residues of the pictured poles lie well above what was set as the numerical accuracy, i.e., that they are not numerical artifacts. The depicted singularities are discretizations of branch cuts with branch points at values of $\xi$ given in Eqs. (16) and (20) as well as at $\xi_{0}+\xi_{ \pm}$and $2 \xi_{ \pm}$(see also Ref. [34] for a related statement based on a smaller data set from the previous version of the present manuscript).

of the analytic continuation of the Borel transform has been argued to disappear once the gradient expansion is supplemented with exponentially decaying terms,

$$
\delta \mathcal{A} \sim e^{-\xi_{0} w},
$$

where $\xi_{0}$ denotes the beginning of a cut in the complex Borel plane. In the case under consideration, the cut closest to the origin starts, up to five decimal places, at

$$
\xi_{0}=1.5000 / \gamma
$$

The constants $\xi_{0}$ appearing in Eq. (15) can in general be complex (they then come in conjugate pairs), and in the examples analyzed so far in the literature, they appear in positive integer multiples. Each such term comes with an infinite gradient expansion of its own, and the term with the lowest $\xi_{0}$ in a given family comes with an independent complex integration constant. In all known cases $[15,21$, 52-54] (see Ref. [1] for a review), those least-damped modes within a given family coincide with singularities of retarded equilibrium two-point functions of the energy-momentum tensor at vanishing momentum.

In the context of the RTA kinetic theory, the studies of Ref. [22] reveal the presence of a zero-momentum fast-evolving mode in the isotropization of the energymomentum tensor to its equilibrium form:

$$
\delta\left\langle T^{\mu \nu}\right\rangle \sim e^{-\omega_{0} T t}, \quad \omega_{0}=1 / \gamma .
$$


The nontrivial background flow is known to modify the above equation to the form $[15,57,58]$

$$
\delta\left\langle T^{\mu \nu}\right\rangle \sim e^{-\omega_{0} \int T(x) u_{\mu} d x^{\mu}},
$$

which for the case of Bjorken flow, neglecting subleading terms at large values of $w$, reduces to

$$
\delta \mathcal{A} \sim e^{-\frac{3}{2} \omega_{0} w} .
$$

This, together with Eq. (17), reproduces Eq. (16). Let us also note here that the analysis in Ref. [59] reveals that the cut along the real axis seen in Fig. 1 must be, in fact, an infinite collection of independent cuts. They all start at the same branch point, i.e., $\xi=\xi_{0}$, but are characterized by different discontinuities and are interpreted as an infinite set of modes carrying information about the initial distribution function to late times $w$.

Importantly, Fig. 1 contains also a pair of singularities characterized by

$$
\xi_{ \pm} \approx(2.25016 \pm 1.29898 i) / \gamma .
$$

If one were to apply straightforwardly the lessons from earlier studies of other models of expanding plasmas, these complex values of $\xi_{0}$ would be interpreted as oscillatory-type transient contributions to the pressure anisotropy. As shown in the next section, in which we argue that these are unphysical, this natural-looking conclusion is premature. This is an important point, since such singularities appear also in other kinetic theory models, such as those with $\tau_{\text {rel }} \sim T^{-\Delta}$, for $0<\Delta<3$. Notably, for $\Delta>2$, the unphysical modes are actually closest to the origin, so naively they would correspond to the dominant nonhydrodynamic corrections [59].

\section{ANALYTIC PROPERTIES OF RTA KINETIC THEORY}

To explain the singularities of the Borel transform at $\xi_{ \pm}$, see Eq. (20) and Fig. 1, we use the integral equation [49], which follows from the Boltzmann equation [48] and directly determines the local energy density $\mathcal{E}(\tau)$ :

$$
g(\tau)=\mathcal{E}_{0}(\tau)+\frac{1}{2} \int_{\tau_{0}}^{\tau} \frac{\mathrm{d} \tau^{\prime}}{\tau_{\text {rel }}\left(\tau^{\prime}\right)} H\left(\frac{\tau^{\prime}}{\tau}\right) g\left(\tau^{\prime}\right) .
$$

In the expression above, $\mathcal{E}_{0}(\tau)$ carries information about initial conditions but will not be relevant in the following analysis. The object of interest is the energy density $\mathcal{E}(\tau)$, which appears in

$$
g(\tau)=\mathcal{E}(\tau) e^{\int_{\tau_{0}}^{\tau} \frac{\mathrm{d} \tau^{\prime}}{\operatorname{rel}\left(\tau^{\prime}\right)}}
$$

as well as in $\tau_{\text {rel }}(\tau)$ through Eqs. (3) and (4). The function $H(q)$ originates from the second moment of the equilibrium distribution function, Eq. (1), and reads

$$
H(q)=q^{2}+\frac{\arctan \sqrt{\frac{1}{q^{2}}-1}}{\sqrt{\frac{1}{q^{2}}-1}} .
$$

What will be crucial in the following is the analytic structure of $H$. Since the natural variable in our considerations is $w$ and at late times $w \sim \tau^{2 / 3}$, we shall write the argument of $H$ as $q=\left(w^{\prime} / w\right)^{3 / 2} \equiv \zeta^{3 / 2}$.

Among the singularities of $H\left(\zeta^{3 / 2}\right)$, the one of interest is the branch point stemming from the square root in the denominator. It appears as a singularity when the arctan function in the numerator is taken in a nonprincipal branch. As a result, one finds

$$
H\left(\zeta^{3 / 2}\right) \sim\left(1-\zeta^{3}\right)^{-1 / 2},
$$

which has branch points at third roots of unity. We will be interested in the ones located at

$$
\zeta_{ \pm}=e^{ \pm i \frac{2}{3} \pi}
$$

The key observation is that the presence of singularities in the complex $\zeta$ plane leads to inequivalent choices of integration contours in Eq. (21), the only physical choice being homologous to the integration along the real axis. If one uses the late-time solution for $g(\tau(w))$ obtained from Eq. (11) under the integral in Eq. (21) and considers two inequivalent contours around $\zeta_{-}$(or, similarly, $\zeta_{+}$), denoted $\mathcal{C}_{1}$ and $\mathcal{C}_{2}$, one finds

$$
\delta g(w) \sim\left[\int_{\mathcal{C}_{1}} \mathrm{~d} \zeta-\int_{\mathcal{C}_{2}} \mathrm{~d} \zeta\right] e^{\frac{3 w}{2 \gamma} \zeta} H\left(\zeta^{3 / 2}\right) \times \cdots,
$$

where the ellipsis denotes terms subleading at large $w$. Similarly to the analysis around Eq. (14), the branch cuts lead to contributions to $\delta g$ of the form

$$
\delta g \sim e^{-\frac{3 \zeta \pm}{2 \gamma} w},
$$

where we truncated subleading terms at large values of $w$. Finally, note that $\delta g$ and $\delta \mathcal{E}$ are related through Eq. (22) with, at late times/large values of $w, e^{\int_{\tau_{0}}^{\tau} \frac{\mathrm{d} \tau^{\prime}}{\operatorname{rel}\left(\tau^{\prime}\right)}} \sim e^{\frac{3}{2 \gamma} w}$, which ultimately gives

$$
\delta \mathcal{E}_{ \pm} \sim e^{-\frac{3}{2 \gamma}\left(\zeta_{ \pm}-1\right) w} .
$$

Comparing the exponent in the equation above with Eq. (20), one observes a remarkable agreement up to four decimal places. As a further way of corroborating this result, one can use the techniques utilized earlier in this context in Refs. $[15,21]$ to match the square root character of the branch cut in Eq. (24) with the leading power-law correction in $w$ to contributions from the $\xi_{ \pm}$singularities in the Borel plane, with very good agreement.

All this taken together gives us confidence that the correct interpretation of the singularities given in Eq. (20) is that they correspond not to physical excitations but 
rather to analytic properties of kinetic theory for complexified values of the $w$ variable. The physical integration contour in Eq. (21) along real values of $\tau^{\prime}$ does not pick up these contributions (and, no wonder, they are not seen in solutions of the initial value problem discussed in Ref. [59]). The gradient expansion itself does not preclude unphysical choices of contour, and this is reflected in its large-order behavior. Similar phenomena can be seen in the integral equation considered in Ref. [60] or in the ghost-instanton story of Ref. [43]. Their origin goes back to the fundamental point of resurgence: all nonperturbative information is encoded in the large-order behavior of the perturbative sector.

\section{HYDRODYNAMIZATION COMPARED}

In previous sections, we have demonstrated that hydrodynamization in the RTA takes place through the decay of transient nonhydrodynamical modes in complete analogy to the situation at strong coupling. On the other hand, the RTA shares structural similarities with EKT-while the collision kernels of the QCD effective kinetic theory have much richer structure than the RTA, many qualitative features at the level of kinetic theory coincide. In this section, we further strengthen the connection between RTA and EKT by noting that the similarity between these two theories goes beyond abstract structural similarity and that they agree semiquantitatively when the values of $\eta / s$ are matched between the two theories.

To demonstrate the extent of quantitative agreement between different models, inspired by Ref. [46], we introduce a new, rescaled variable $\tilde{w} \equiv \frac{w}{4 \pi \eta / s}$. This is useful for such comparisons, since the late-time behavior of the pressure anisotropy $\mathcal{A}$ is given by

$$
\mathcal{A}_{H}(\tilde{w})=\frac{2}{\pi \tilde{w}}+\mathcal{O}\left(\frac{1}{\tilde{w}^{2}}\right)
$$

The leading behavior is completely universal and does not depend on the value of $\eta / s$. Deviations from the asymptotic form characterize contributions arising beyond first-order hydrodynamics, and, indeed, we say that the system has reached the hydrodynamic regime when for a given state the relative difference between $\mathcal{A}$ and $\mathcal{A}_{H}$ remains smaller than some threshold value. Figure 2 shows a comparison of the time evolution of the system evolved in the EKT from Ref. [9], RTA using the methodology of Refs. [48,49], and numerical AdS/CFT calculation of Refs. [13,45,61]. For the EKT and RTA simulations, we took the initial condition used in Ref. [9], whereas for the AdS/CFT simulation, we took typical initial conditions from Ref. [45]. We evolved the systems using EKT with $\lambda=10$ corresponding to $\eta / s \approx 0.642$, holography with $\eta / s=1 / 4 \pi$, and RTA with $\gamma$ fixed to reproduce the value of $\eta / s$ of either model. Note that every kinetic theory curve in Fig. 2 corresponds to a different initial distribution function. In all these models,

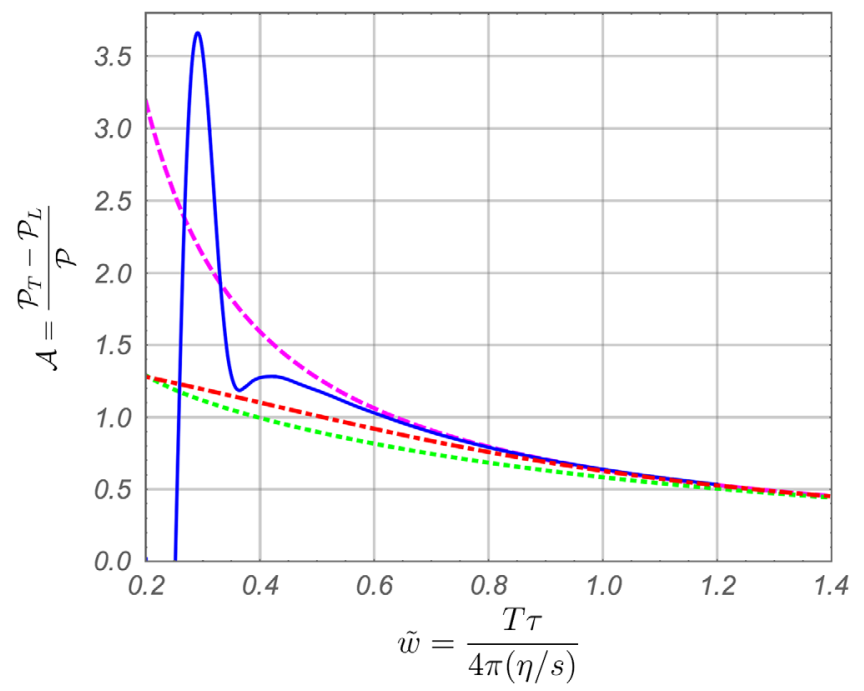

FIG. 2. The dashed magenta curve represents first-order hydrodynamics, the blue line is the holographic result, and the red dashed-dotted line is from EKT. The green dotted curve stands for a solution of RTA starting from initial distribution similar to EKT and with the same shear viscosity, $\eta / s=0.624$. Despite differences in microscopic dynamics, one sees significant qualitative and some quantitative similarities between different theories.

the evolution is similar but distinct. Remarkably, in each case-despite vastly differing microphysics - the evolution converges to first-order viscous hydrodynamics roughly at the same value of $\tilde{w}$ variable, i.e. $\tilde{w} \approx 1$. It is striking that in all these cases the pressure anisotropy at the time of hydrodynamization is as high as $\mathcal{A} \approx 0.6-0.8$.

The structural and quantitative similarities of EKT and RTA suggest that the gradient expansion in EKT also exhibits a zero radius of convergence and that the weak coupling hydrodynamization is driven by the same qualitative process. This is connected with the notion of attractors that have been explored in both RTA and holography $[28,29]$. To what extent these insights translate to EKT is an important problem.

\section{ACKNOWLEDGMENTS}

We would like to thank the authors of Ref. [39] as well as I. Aniceto, G. Dunne, W. Florkowski, R. Janik, P. Romatschke, W. van der Schee, M. Martinez, and M. Strickland for valuable discussions and correspondence. Research at Perimeter Institute is supported by the Government of Canada through Industry Canada and by the Province of Ontario through the Ministry of Research \& Innovation. M. P. H. acknowledges support from the Alexander von Humboldt Foundation and the Federal Ministry for Education and Research through the Sofja Kovalevskaja Award. M. S. and V. S were supported by the Polish National Science Centre Grant No. 2015/19/B/ ST2/02824. 
[1] W. Florkowski, M. P. Heller, and M. Spalinski, Rep. Prog. Phys. 81, 046001 (2018).

[2] P. Romatschke and U. Romatschke, arXiv:1712.05815.

[3] P. B. Arnold, G. D. Moore, and L. G. Yaffe, J. High Energy Phys. 01 (2003) 030.

[4] R. Baier, A. H. Mueller, D. Schiff, and D. T. Son, Phys. Lett. B 502, 51 (2001).

[5] P. B. Arnold, C. Dogan, and G. D. Moore, Phys. Rev. D 74, 085021 (2006).

[6] A. Kurkela and G. D. Moore, J. High Energy Phys. 12 (2011) 044.

[7] A. Kurkela and G. D. Moore, J. High Energy Phys. 11 (2011) 120.

[8] A. Kurkela and E. Lu, Phys. Rev. Lett. 113, 182301 (2014).

[9] A. Kurkela and Y. Zhu, Phys. Rev. Lett. 115, 182301 (2015).

[10] J. M. Maldacena, Adv. Theor. Math. Phys. 2, 231 (1998).

[11] P. M. Chesler and L. G. Yaffe, Phys. Rev. D 82, 026006 (2010).

[12] P. M. Chesler and L. G. Yaffe, Phys. Rev. Lett. 106, 021601 (2011), arXiv:1011.3562.

[13] M. P. Heller, R. A. Janik, and P. Witaszczyk, Phys. Rev. Lett. 108, 201602 (2012).

[14] J. Casalderrey-Solana, H. Liu, D. Mateos, K. Rajagopal, and U. A. Wiedemann, Gauge/String Duality, Hot QCD and Heavy Ion Collisions (Cambridge University Press, Cambridge, England, 2011).

[15] M. P. Heller, R. A. Janik, and P. Witaszczyk, Phys. Rev. Lett. 110, 211602 (2013).

[16] J. Anderson and H. Witting, Physica (Amsterdam) 74, 466 (1974).

[17] A. Buchel, M. P. Heller, and R. C. Myers, Phys. Rev. Lett. 114, 251601 (2015).

[18] R. A. Janik, G. Plewa, H. Soltanpanahi, and M. Spalinski, Phys. Rev. D 91, 126013 (2015).

[19] G. S. Denicol and J. Noronha, arXiv:1608.07869.

[20] R. Baier, P. Romatschke, D. T. Son, A. O. Starinets, and M. A. Stephanov, J. High Energy Phys. 04 (2008) 100.

[21] M. P. Heller and M. Spalinski, Phys. Rev. Lett. 115, 072501 (2015).

[22] P. Romatschke, Eur. Phys. J. C 76, 352 (2016).

[23] A. Kurkela and U. A. Wiedemann, arXiv:1712.04376.

[24] P. Romatschke, Eur. Phys. J. C 75, 305 (2015).

[25] M. Habich, G. A. Miller, P. Romatschke, and W. Xiang, Eur. Phys. J. C 76, 408 (2016).

[26] M. Spalinski, Phys. Rev. D 94, 085002 (2016).

[27] P. Romatschke, Eur. Phys. J. C 77, 21 (2017).

[28] P. Romatschke, Phys. Rev. Lett. 120, 012301 (2018).

[29] M. Spaliński, Phys. Lett. B 776, 468 (2018).

[30] P. Romatschke, J. High Energy Phys. 12 (2017) 079.

[31] M. Strickland, J. Noronha, and G. Denicol, Phys. Rev. D 97, 036020 (2018).
[32] W. Florkowski, E. Maksymiuk, and R. Ryblewski, Phys. Rev. C 97, 024915 (2018).

[33] G. S. Denicol and J. Noronha, Phys. Rev. D 97, 056021 (2018).

[34] J. Casalderrey-Solana, N. I. Gushterov, and B. Meiring, arXiv:1712.02772.

[35] J.-P. Blaizot and L. Yan, Phys. Lett. B 780, 283 (2018).

[36] J. Brewer and P. Romatschke, Phys. Rev. Lett. 115, 190404 (2015).

[37] H. Bantilan, J. T. Brewer, T. Ishii, W. E. Lewis, and P. Romatschke, Phys. Rev. A 94, 033621 (2016).

[38] S. Grozdanov, N. Kaplis, and A. O. Starinets, J. High Energy Phys. 07 (2016) 151.

[39] D. Bazow, M. Martinez, and U. W. Heinz, Phys. Rev. D 93, 034002 (2016); 93, 034002 (2016).

[40] M. Martinez and U. Heinz (private communication).

[41] G. V. Dunne, Lectures given at the Schladming Winter School 2015, Schaldming, Austria (unpublished).

[42] G. V. Dunne and M. Ünsal, Proc. Sci., LATTICE2015, (2016) 010.

[43] G. Basar, G. V. Dunne, and M. Unsal, J. High Energy Phys. 10 (2013) 041.

[44] I. Aniceto, G. Başar, and R. Schiappa, arXiv:1802.10441.

[45] J. Jankowski, G. Plewa, and M. Spalinski, J. High Energy Phys. 12 (2014) 105.

[46] L. Keegan, A. Kurkela, P. Romatschke, W. van der Schee, and Y. Zhu, arXiv:1512.05347.

[47] J. Bjorken, Phys. Rev. D 27, 140 (1983).

[48] G. Baym, Phys. Lett. 138B, 18 (1984).

[49] W. Florkowski, R. Ryblewski, and M. Strickland, Phys. Rev. C 88, 024903 (2013).

[50] See Supplemental Material at http://link.aps.org/ supplemental/10.1103/PhysRevD.97.091503 for details about 425 lowest terms in the hydrodynamic gradient expansion, as defined by Eq. (8).

[51] A. Jaiswal, Phys. Rev. C 87, 051901 (2013).

[52] A. Buchel, M. P. Heller, and J. Noronha, Phys. Rev. D 94, 106011 (2016).

[53] G. Basar and G. V. Dunne, Phys. Rev. D 92, 125011 (2015).

[54] I. Aniceto and M. Spaliński, Phys. Rev. D 93, 085008 (2016).

[55] H. S. Yamada and K. S. Ikeda, arXiv:1308.4453.

[56] W. Florkowski, R. Ryblewski, and M. Spalinski, Phys. Rev. D 94, 114025 (2016).

[57] R. A. Janik and R. B. Peschanski, Phys. Rev. D 74, 046007 (2006).

[58] M. P. Heller, R. A. Janik, M. Spaliński, and P. Witaszczyk, Phys. Rev. Lett. 113, 261601 (2014).

[59] M. P. Heller and V. Svensson, arXiv:1802.08225.

[60] I. Aniceto, J. Phys. A 49, 065403 (2016).

[61] M. P. Heller, R. A. Janik, and P. Witaszczyk, Phys. Rev. D 85, 126002 (2012). 